\title{
3 Research Square

\section{Management of multiple rib fractures-results from a Major Trauma Centre with review of the existing literature}

Debkumar Chowdhury ( $\nabla$ dc7740@my.bristol.ac.uk)

Royal Preston Hospital https://orcid.org/0000-0002-4064-2257

\section{P. Okoh}

Specialty Registrar, Emergency Medicine, Emergency Department, Royal Preston Hospital

H. Dambappa

Specialty Registrar, Emergency Medicine, Emergency Department, Royal Preston Hospital

\section{Short Report}

Keywords: Rib fracture, Pain Management, Trauma

Posted Date: December 1st, 2020

DOl: https://doi.org/10.21203/rs.3.rs-118238/v1

License: (c) (i) This work is licensed under a Creative Commons Attribution 4.0 International License.

Read Full License 


\section{Abstract}

Introduction Rib fractures are amongst the most common fractures following major trauma presenting to the Emergency Department. It accounts for more than $15 \%$ of ED presentations ${ }^{(1)}$ on a global scale. As the population ages the incidence of rib fractures also rises often following falls from a relatively small height being part of fragility fractures. The impact of rib fractures is even more significant in the patient with underlying chronic respiratory conditions.

Aim To assess our current management of rib fractures at our trauma centre

Method We collected our data from the TARN Registry primarily focussing on patients with multiple rib fractures. The main components were the analgesic requirement of our patients. We also studied the number of rib fracture stabilisation procedures and the average number of ribs fixed.

Results The data was collected retrospectively over a period of 12 months. There were $\mathbf{3 1 3}$ patients identified as having chest wall injuries. From the data, $41.9 \%$ (131) of patients were over the age of 65 years. A significant proportion of our patients $\mathbf{3 4 . 5 \%}$ (108) were noted to have multiple rib fractures ( $>3$ Ribs). It was noted that $\mathbf{3} \%$ (9) of the 313 patients required operations. The average of the patients included in the study was noted to be 61 years with an age range of 17-92 years.

Conclusion The mainstay management of rib fracture is provision of adequate analgesia and the prevention of respiratory complications that can all stem from poor ventilatory function amongst other patient factors and injury patterns. Through the decades, surgical stabilisation has gained pace and has found its niche in the management of rib fractures.

\section{Introduction}

The main respiratory complications that arise from fractured ribs is multifactorial. It is well known that inadequate pain management in these patients lead to complications arising from poor ventilatory function. With an already compromised underlying pulmonary system this compounds on the effect on an already failing system leading to significant pulmonary complications. Rib fractures in the form of flail segments pose a particular problem in this regard. When one considers an elderly patient with limited Functional Residual Capacity with an underlying diagnosis of chronic obstructive airway disease, with the addition of the presence of a flail segment will invariably lead to respiratory embarrassment either acutely and /or lead to pulmonary complications in the form of basal atelectasis and pneumonia.

The main determinants of the rib score generally underpins the number of ribs that are fractured as well as the age of the patient directly correlate with morbidity and mortality. The traditional rib score is the product of the number of breaks and the sides with the addition of the age factor. Our rib score is more detailed and encompasses several other variables that have are thought to indicate a more accurate picture of the mortality and morbidity. It is recognised that if there are four or more fractures that are associated with a higher mortality rate and if there ae 7 or more fractures then mortality rate can be as 
high as $29 \%$ In the presence of flail segment, the mortality rate is noted to be higher at $33 \%$ perhaps as due to further respiratory embarrassment stemming from the paradoxical movement of the ribs on respiration (2).

\title{
Method
}

The data collected from the TARN registry was over a consecutive period of 12 months, studying patients with rib fractures. The inclusion criteria for the study were all patients with rib fractures irrespective of whether it was the primary or secondary diagnosis. The individual patients were studied and subgroup analysis was done on rib fixation. The average number of ribs fixed were also studied with the length of stay in hospital included in the analysis. In addition, the analgesic requirement of the individual patients was also studied and tabulated according to the needs. We noted that there was a overlap in patients receiving epidural and the group receiving opioids. Further subgroup analysis would be a feature in future articles comparing the effect on the individual analgesic modality.

\section{Results}

Of the 9 patients that required chest fixations, the ISS score is noted to be within the high severity between 16-25. This indicates that the most severely injured patients required operative management of their chest wall injuries.

Both the groups of Intravenous opioids and PCA highlight the same group of patients.

The data was collected retrospectively over a period of 12 months. There were $\mathbf{3 1 3}$ patients identified as having chest wall injuries. From the data, $41.9 \%$ (131) of patients were over the age of 65 years. A significant proportion of our patients $\mathbf{3 4 . 5 \%}$ (108) were noted to have multiple rib fractures ( $>3$ Ribs). It was noted that $3 \%$ (9) of the 313 patients required operations. The average of the patients included in the study was noted to be 61 years with an age range of 17-92 years. From the data analysis it is clear that the cases that underwent operative fixation had on average 4-5 ribs. This data is compatible with the preexisting studies that demonstrate a similar clinical picture.

\section{Discussion}

\author{
Traditional Rib Score ${ }^{(3)}$
}

\section{Rib fracture Score= $($ Breaks $\times$ Sides $)+$ Age Factor}


Rib Score used in our Trust ${ }^{(4)}$

Age +1 for each 10 years from aged 10

Ribs +3 for each individual fracture

Chronic lung disease +5

Existing anti-platelet or anti-coagulant use +4

SaO2 +2 for each $5 \%$ decrease in saturation below $95 \%$

Outcome of scoring

Score $0-10$ = CONSERVATIVE

Simple oral analgesia

? safe to discharge home. Review in the knowledge of age and comorbidities.

If pain persists or increases add in a PCA

Score 11-20 = PROGRESSIVE

PCA +/- adjuncts

If unable to take deep breaths or cough consider an epidural or paravertebral catheter

Score 21-30 = AGGRESSIVE

Epidural or paravertebral catheter

Also consider interpleural and intercostal blocks

If CPAP indicated for respiratory support level two bed required 
Score $>31=$ EMERGENT

Epidural or paravertebral catheter

Do not site epidural in a ventilated patient until they are suitable for extubation

As it can be seen that the rib score that we use in our Trust is more detailed and incorporates several important patient characteristics that has a direct correlation to patient outcomes. As explained earlier that if the patient has underlying chronic lung disease, any traumatic injuries to the chest would lead to impaired ventilator function leading to complications. The importance of anti-platelet or anticoagulant use would signify if there were concerns about underlying haemothorax/ bleeding from the fractured ribs. Reduced oxygen saturations acutely would directly correlate with inadequate oxygenation and would indirectly indicate that ventilation to the poorly aerated parts of the lung would be compromised. Having a more detailed scoring system and associated management plan could potentially support the less experienced clinician. The true impact of the introduction of the revised rib fracture scoring system remains to be seen. The implementation of this tool in the Emergency Department will hopefully optimise the pain management of the patients earlier on as well as identify the 'at risk' patients.

The optimisation of analgesia in rib fracture patients is challenging due to varied response to analgesia bearing in mind of the patients underlying co-morbidities. The associated respiratory depression with the use of intravenous can be significant and needs to be taken into consideration when administering the same in a patient with impaired ventilatory function. In the review article by May ${ }^{(5)}$ et al., the various regional block techniques have been described and these have proved to be useful in clinical practice. It is perhaps the early recognition that pain has a significant physiological effect on patient's wellbeing and eventually outcomes.

\section{Optimal management of rib fractures}

The surgical management of rib fractures have been debatable and there is often a shared responsibility between orthopaedic, traumatologist and cardio-thoracic surgeons. In the United Kingdom, orthopaedic surgeons undertake rib stabilisation. There have been studies ${ }^{(6)}$ that have suggested that a combination of multiple rib fractures and underlying pulmonary contusions would be a contraindication for operative intervention. There also suggestions that patients with severe pulmonary contusions would not be suitable candidates for surgical fixation. However, currently there are no imaging modality that can accurately grade the degree of pulmonary contusion. 
A comprehensive meta-analysis done by Tohidi et al ${ }^{(7)}$ noted that there was a significant reduction in the risk of death in the surgical fixation group with also reduced need for tracheostomy and chest wall deformity. The main confounding factor that the authors identified in the studies available were the varying indications for the procedure.

Indications for operative management ${ }^{(8)}$

Recommended: $₫$

- $\geq 5$ rib flail chest requiring mechanical ventilation;

- Symptomatic non-union;

- Severe displacement found during a thoracotomy for another reason.

Consider:

- $\geq 3$ rib flail not requiring mechanical ventilation;

- $\geq 3$ ribs with severely displaced fractures (bi-cortical displacement);

- $\geq 3$ ribs with mild to moderate displacement and $50 \%$ reduction of expected forced vital capacity percent despite optimal pain management.

Absolute contraindications: Contaminated field.

Relative contraindications:

- Severe lung contusion requiring prolonged mechanical ventilation;

- High cervical spine injury requiring mechanical ventilation.

Pain scores in isolation are not currently being considered ${ }^{(9)}$ as an indication for operative intervention due to the subjectivity and the patient-to patient variability.

One of the main domains of trauma care that is poorly studied is the follow-up period. Once the patient has had their immediate management and subsequently admitted/transferred for rehabilitation there is usually a loss to follow-up. Not including these results in an incomplete data set and the true impact of any intervention. The length of time and the frequency of follow up is a matter of debate with some authors purporting a longer period of follow up with a reduced frequency to gain a true understanding of the intervention. 
Historically, there had been an increased uptake of patients undergoing fixation of rib fractures in patients with flail segments in the 1950s in patients with respiratory failure. However, with the increase in uptake of positive pressure ventilation the need for open reduction had reduced. It is only recently that there has been renewed interest in open reduction and internal fixation in patients with multiple rib fractures. Trials performed recently had shown significant improvement in various parameters ${ }^{(10)}$. This ranges from the incidence of respiratory infections to length of stay in ICU. One of the main differences was the improvement in forced vital capacity in patients undergoing surgical intervention compared to those that do not. The number of ribs fixed ranged commonly between 4-6 ribs in our study this was related to the anatomical position of the fracture and accessibility to the fractures as not all ribs fractured need to be fixed ${ }^{(11)}$. It is important that the site is accessed whereby any residual haemothorax can be drained and any empyema drained appropriately. It may be postulated that as the population increases in countries like the United Kingdom there will be an increase in uptake in rib fracture stabilisation procedures. The benefit of the operative procedure in patients with less than 5 rib fractures remains to be seen.

\section{Future studies}

The reader would agree that this indeed a large study which raises the scope for detailed in-depth analysis of various factors. We have identified that the individual mechanism of injuries that lead to rib fractures can be studied and appropriate primary prevention management strategies implemented. Through this article we hope the reader would appreciate the scope for further study comparing the traditional rib score with the new proposed rib fracture score. We have appreciated the fact that the traditional rib fracture score does not incorporate the significant factors that significantly contribute to the outcome on the overall rib fracture management.

Through this article we sincerely hope that the orthopaedic surgeons/cardiothoracic surgeons would consider operative interventions in patients that have significant rib fractures but not necessarily meet the current proposed criteria. With time we hope that the surgeons would have a lower threshold for operative intervention to maximise patient outcomes. Larger multicentric randomised controlled trials are needed to demonstrate the difference in outcomes in these two groups for a comparative analysis.

\section{Conclusion}

The mainstay management of rib fracture is provision of adequate analgesia and the prevention of respiratory complications that can all stem from poor ventilatory function amongst other patient factors and injury patterns. Through the decades, surgical stabilisation has gained pace and has found its niche in the management of rib fractures.

\section{Declarations}




\section{Acknowledgment}

The authors would like this opportunity to thank the patients and the staff involved in the care of the severely injured patients included in our study

\section{Conflict of Interest}

The authors have not highlighted any conflict of interest during the course of the study and in the write up of this article.

\section{Ethical Statement}

There were no ethical concerns identified during the course of the study.

\section{Funding}

There was no funding applied to undertake this study.

\section{References}

1) Battle et al. Predicting outcomes after blunt chest wall trauma: development and external validation of a new prognostic model. Crit Care 2014, 18:R

2) Holcomb J, McMullin N, Kozar R, Lygas M, Moore F. Morbidity from rib fractures increases after age 45. J Am Coll Surg 2003; 196: 549-55 5.

3) Nirula R, Diaz J, Trunkey D, Mayberry J. Rib fracture repair: indications, technical issues, and future directions. World J Surg 2009; 33: 14-22

4) Sr G. Nixon Dr E Baird et al. Acute Pain Management for adult patients with fractured ribs Ref 9/2017.

5) L May, MBChB FRCA MAcadMEd, C Hillermann, MBChB FRCA DA(SA), S Patil, MS MSc FRCSEd (T\&O), Rib fracture management, BJA Education, Volume 16, Issue 1, January 2016, Pages 26-32,

6) He Z, Zhang D, Xiao H, et al. The ideal methods for the management of rib fractures. J Thorac Dis. 2019;11(Suppl 8):S1078-S1089. doi:10.21037/jtd.2019.04.109

7) Tohidi M., O'Sullivan D., Groome P., and Yach J. D Surgical fixation of multiple rib fractures and the flail chest: A Meta-analysis and review of evidence: Orthopaedic Proceedings 2020 102-B: SUPP_7, 47-47

8) de Moya M, Nirula R, Biffl W Rib fixation: Who, What, When? Trauma Surgery \& Acute Care Open 2017;2: e000059. doi: 10.1136/tsaco-2016-000059

9) de Moya M, Bramos T, Agarwal S, Fikry K, Janjua S. Pain as an indication for rib fixation: a biinstitutional pilot study. J Trauma 2011; 71:1750-4. 
10) Tanaka H, Yukioka T, Yamaguti Y. Surgical stabilization of internal pneumatic stabilization? A prospective randomized study of management of severe flail chest patients. J Trauma 2002; 52:727-32

11) Nickerson TP, Thiels CA, Kim BD, Zielinski MD, Jenkins DH, Schiller HJ. Outcomes of complete versus partial surgical stabilization of flail chest. World J Surg 2016; 40:236-41

\section{Figures}

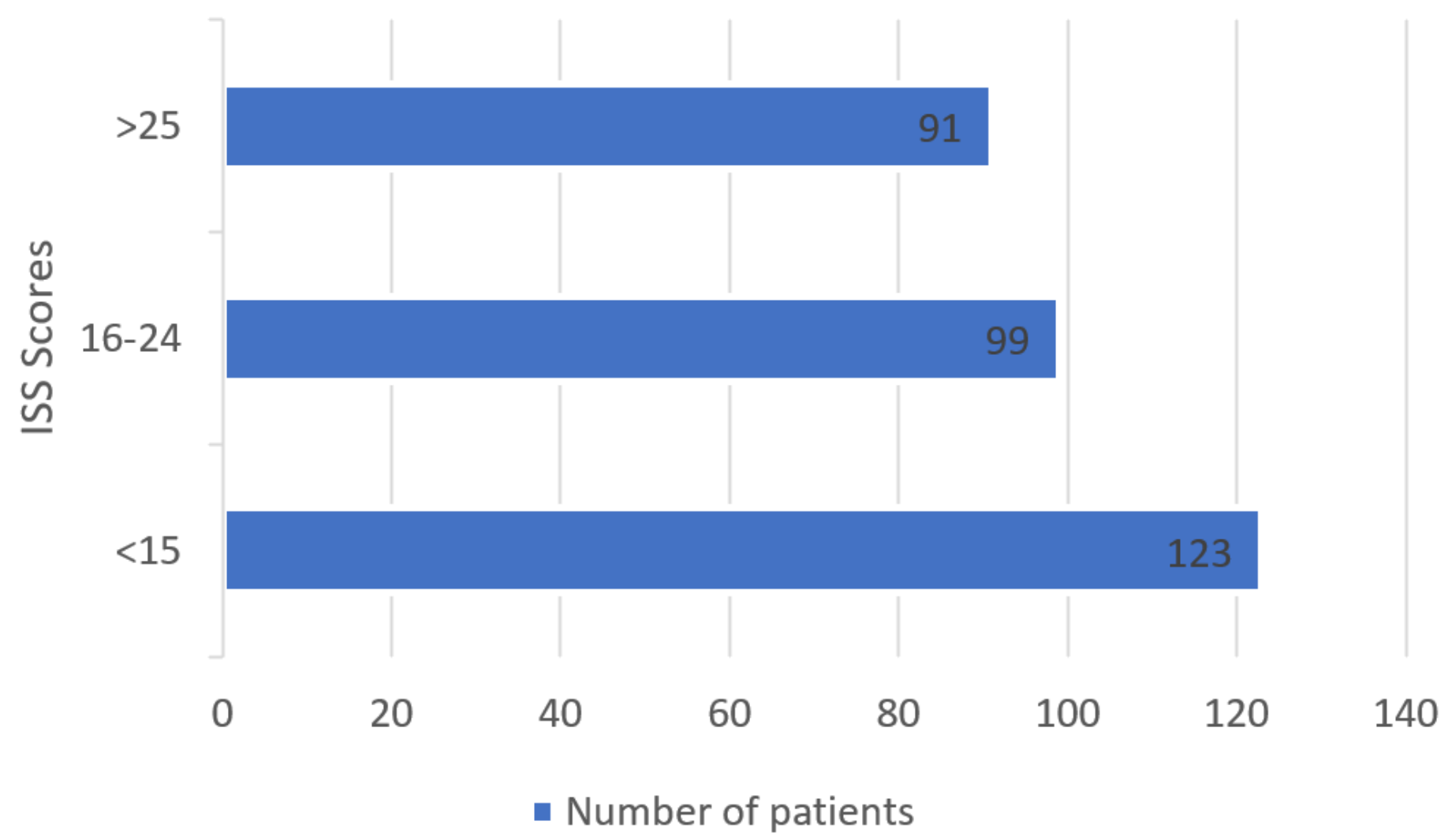

\section{Figure 1}

Number of patients included in the study and their ISS. 


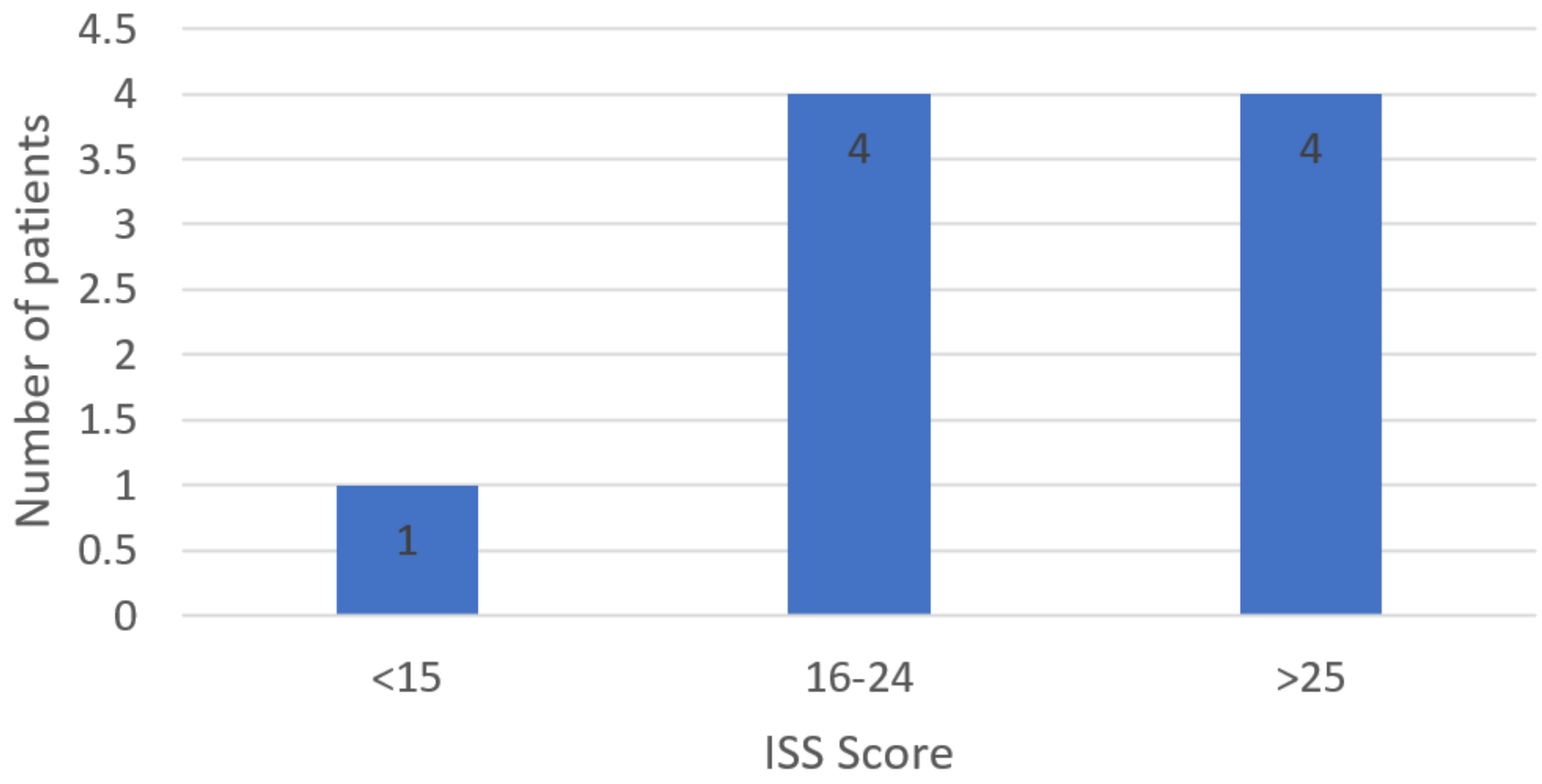

Figure 2

The number of patients requiring chest wall fixations and their ISS

\section{Number of ribs fixed}

Fracture ribs with unilateral flail 3-5 ribs

Fracture ribs with unilateral flail 3-5 ribs

Fracture ribs with unilateral flail $>5$ ribs

Fracture ribs with unilateral flail $>5$ ribs

Fracture ribs with bilateral flail

Fracture ribs with unilateral flail $>5$ ribs

Fracture ribs with unilateral flail $>5$ ribs

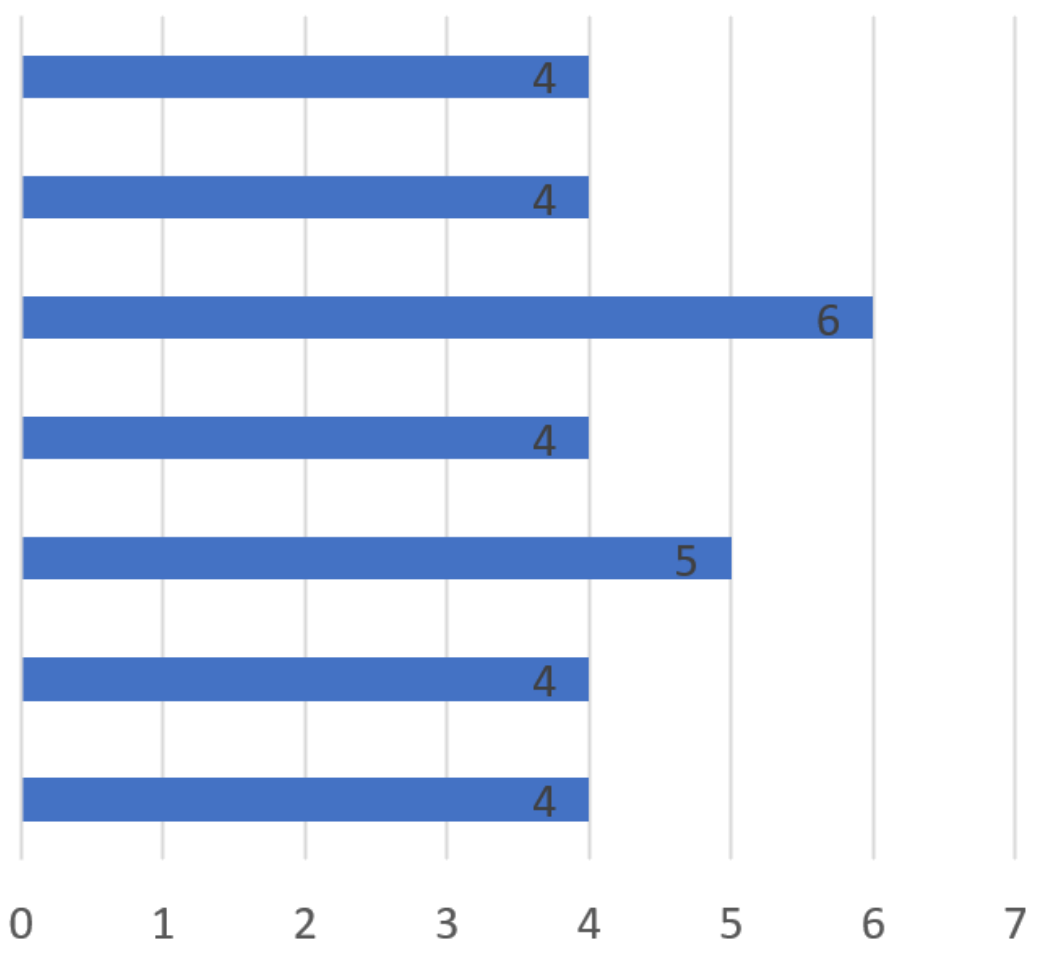

Figure 3 
The number of ribs fixed and their indication

\section{LOS}

60

50

40

30

20

10

0
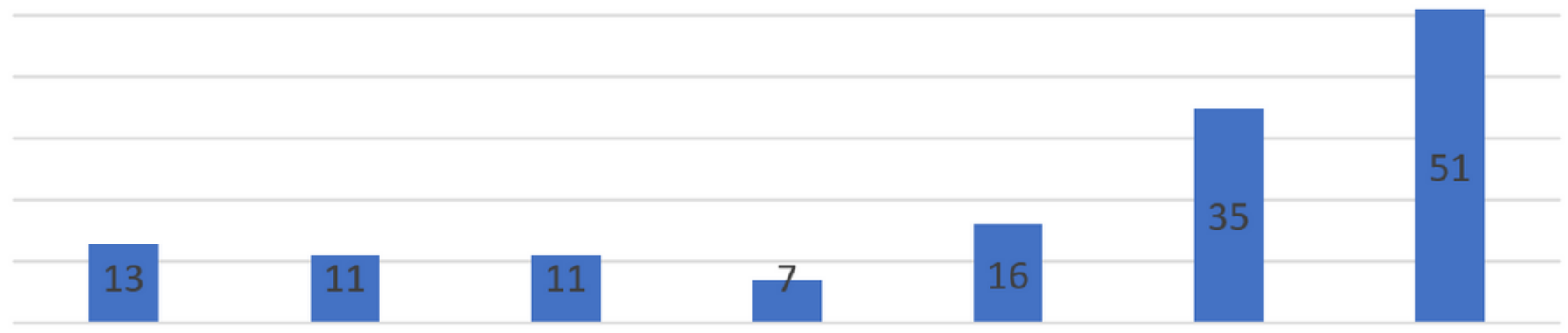

Fracture Fracture Fracture ribs Fracture ribs Fracture ribs Fracture Fracture ribs ribs with ribs with with with with with unilateral unilateral bilateral flail unilateral unilateral unilateral unilateral flail $>5$ ribs flail $>5$ ribs flail $>5$ ribs flail $>5$ ribs flail 3-5 ribs flail 3-5 ribs

Figure 4

Indications and Length of stay in days (LOS)

\section{Number of Patients}

Number of Patients

\section{6}

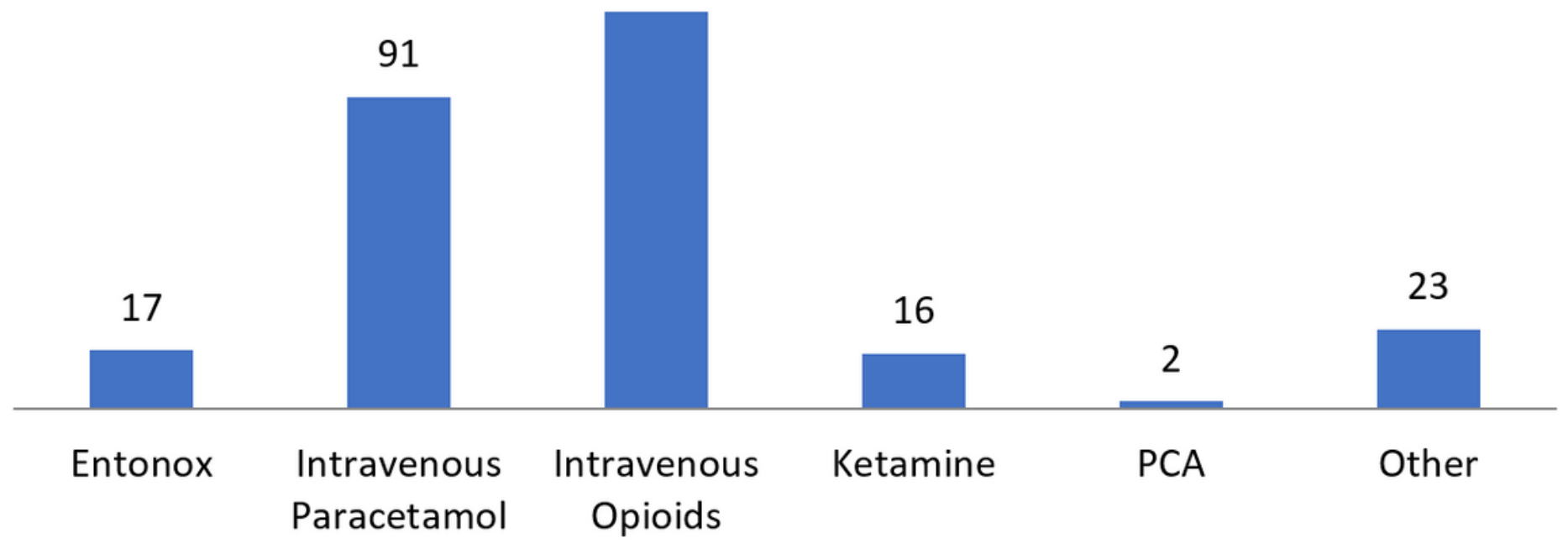

Figure 5

The different analgesic modalities 


\section{Multiple Rib Fracture Pain Management Algorithm}

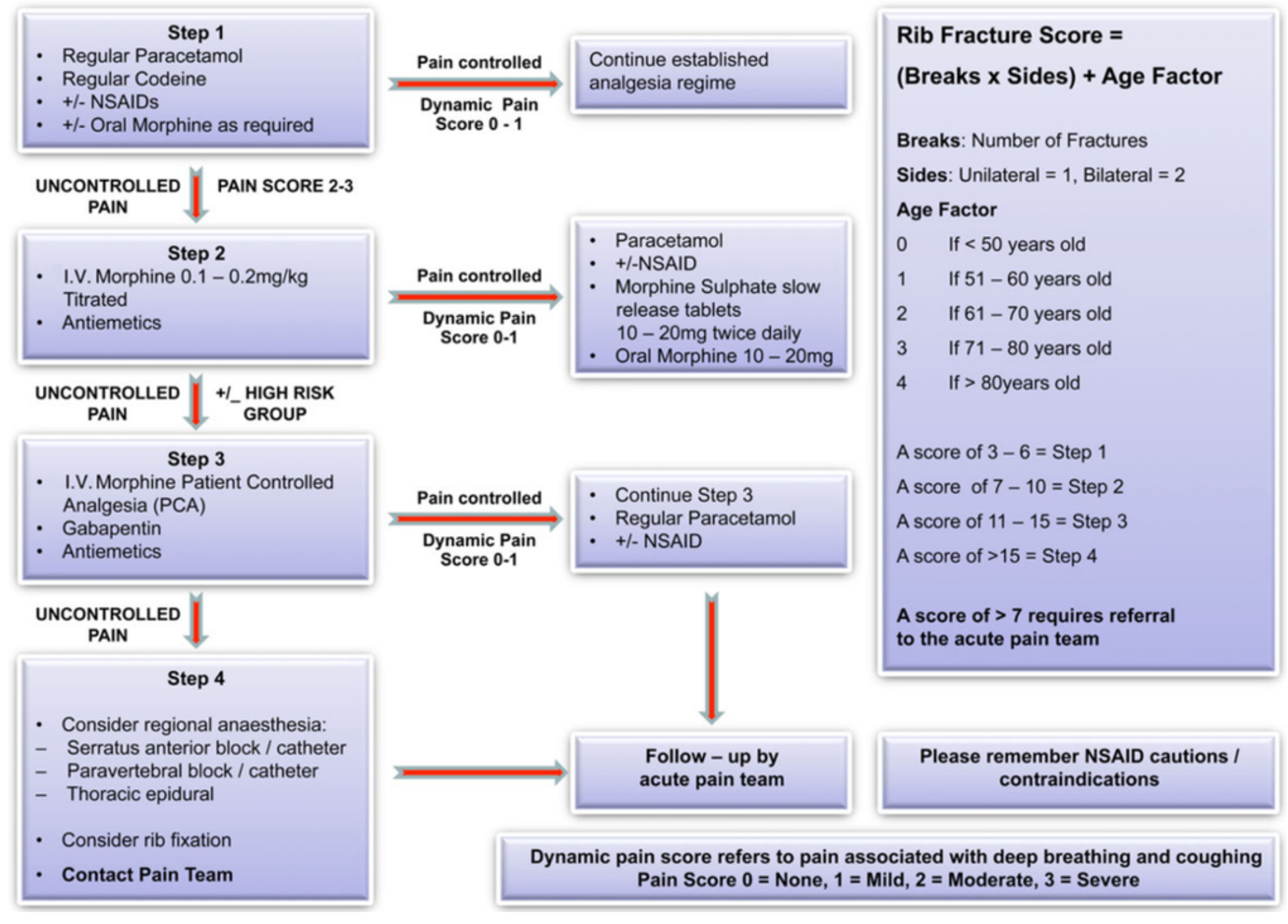

\section{Figure 6}

The traditional multiple rib fracture pain management algorithm 